\author{
(C) К.А. Кузьмичев
}

Самарский государственный медицинский университет, Самара, Российская Федерация

Бесплодие представляет собой важную медико-социальную проблему. Рост числа бесплодных пар приводит к увеличению частоты применения вспомогательных репродуктивных технологий (ВРТ), что выражается в увеличении количества детей, рожденных с применением этих методов. В литературе имеются сведения, раскрывающие особенности заболевания таких детей в неонатальном и раннем постнатальном периодах, демонстрируя значительно более низкие показатели здоровья в сравнении с детьми, рожденными в результате спонтанной беременности. Вопрос о долгосрочном состоянии здоровья детей, рожденных с применением ВРТ все еще является дискуссионным. Сравнительно недавно появились первые крупные мета-анализы, позволяющие достоверно оценить заболеваемость детей, рожденных с помощью ВРТ. В настоящем обзоре представлены данные, обобщающие имеющиеся актуальные сведения по вопросу заболеваемости детей, рожденных с применением ВРТ. Показано отсутствие достоверной разницы в уровне возникновения опухолевых процессов, развития метаболических и психиатрических заболеваний. Наблюдается увеличенный риск возникновения сосудисто-эндотелиальной дисфункции, артериальной гипертонии, сердечно-сосудистых заболеваний. Противоречивые сведения получены в отношении влияния методов ВРТ на репродуктивную функцию: наблюдается снижение качества эякулята у мальчиков, рожденных с применением интрацитоплазматической инъекции сперматозоидов. Представленные сведения позволяют сформировать представление по данному вопросу, однако, в рамках общей заболеваемости. Понозологическая характеристика и определение частоты возникновения отдельных заболеваний все еще представляется актуальной задачей и требует проведения отдельных масштабных проспективных исследований и наблюдения за детьми, рожденными с применением ВРТ на протяжении всей жизни, для установления более точных взаимосвязей.

Ключевые слова: бесплодие; ВРТ; ИКСИ; сердечно-сосудистые заболевания; сахарный диабет; опухоли.

\title{
CHARACTERISTICS OF MORBIDITY OF CHILDREN BORN USING ASSISTED REPRODUCTIVE TECHNOLOGIES
}

\author{
K.A. Kuzmichev
}

Samara State Medical University, Samara, Russian Federation

Infertility is an important medical and social problem. Increasing numbers of infertile couples lead to an increase in the use of assisted reproductive technologies (ART), which is reflected in the increasing number of children born using these methods. Literature provides information on the 
specific features of neonatal and early postnatal diseases in such children, showing significantly lower health parameters compared to children born in result of spontaneous pregnancy. The issue of the long-term health status of children born after ART is still controversial. The first major meta-analyses have appeared quite recently permitting to reliably assess the morbidity of children born after ART. This review presents evidence that summarizes the current data on the morbidity of children born after ART. It shows the absence of a reliable difference in the level of occurrence of neoplastic processes, development of metabolic and psychiatric diseases. There is an increased risk of vascular-endothelial dysfunction, arterial hypertension, cardiovascular diseases. Contradictory data were obtained on the impact of ART methods on reproductive function: a decrease in ejaculate quality was observed in boys born after intracytoplasmic sperm injection. The presented data make it possible to form an idea on this issue, however, within the general morbidity framework. Nosological characteristics and the determination of incidence of certain diseases still remain a relevant task and require special large-scale prospective studies and observation of children born after ART throughout their lives to establish more accurate correlations.

Keywords: infertility; assisted reproductive techniques; intracytoplasmic sperm injections; cardiovascular diseases; diabetes mellitus; neoplasms.

Более шести миллионов детей во всем мире родились в результате применения вспомогательных репродуктивных технологий (ВРТ), порядка 2-3\% родов происходят в европейском регионе после ВРТ. Лечение бесплодия включает в себя применение таких лекарственных средств, как кломифен, фолликулостимулирующий гормон, лютеинизирующий гормон, хорионический гонадотропин человека, прогестерон. При проведении курса лечения бесплодия созревающие яйцеклетки, а затем и эмбрион подвергается воздействию in vivo названных соединений. Такие воздействия приходятся на критические периоды созревания яйцеклеток, преимплантационного и раннего постимплантационного развития, что не позволяет однозначно исключить вклад названных факторов на формирование здоровья детей [1].

Последние три десятилетия происходит детальное изучение потенциальных перинатальных рисков здоровья детей, рожденных с помощью ВРТ. В настоящее время сообщается об уменьшении числа неблагоприятных исходов, связанного с сокращением числа многоплодных родов $[2,3]$. Вместе с тем, необходимо изучать долгосрочные последствия для здоровья детей, в виду вероятности увеличенного риска соматической заболеваемости в детском возрасте по сравнению с детьми, рожденными после спонтанно наступившей беременности [4].

В виду того, что при применении ВРТ могут возникать геномные и эпигеномные нарушения эмбриона, возрастает частота возникновения болезней импринтинга, таких как синдром Беквита-Видемана, синдром Ангельмана, синдром Рассела-Сильвера, некоторые из которых, наряду с другими аномалиями развития включают в себя появление неоплазии например, опухоль Вильмса, гепатобластома при синдроме Беквита-Видеман [5].

В опубликованном в 2017 году исследовании на протяжении 15 лет изучалась заболеваемость детей, рожденных с помощью ВРТ, различными видами опухолевых заболеваний [6]. Было отмечено, что общая частота возникновения неоплазий не превосходит таковую в контрольной группе. Что касается отдельных нозологий, было установлено, что риск развития гепатобластомы был значительно выше в группе детей, рожденных с помощью ВРТ, и был ассоциирован с низкой массой тела при рождении [7]. Мета-анализ, опубликованный в 2020 году, обобщающий данные крупных исследований, проведенных в Великобритании, США, Швеции и др. также подтвердил отсутствие повышенного риска возникновения новообразований в целом у детей, рожденных с применением 
ВРТ, однако, отдельные типы опухолей могут чаще встречаться в данной группе детей, но требуют более целенаправленного исследования [8].

Риск возникновения сердечно-сосудистых и метаболических заболеваний у детей, рожденных с помощью ВРТ, представляется достаточно сложным для оценки. Представленные в литературе исследования основаны на данных по небольшим когортам. С другой стороны, сердечно-сосудистые и метаболические заболевания в основном встречаются среди взрослых, в то время как среди подростков и детей такие заболевания обычно не распространены.

Недавние систематические обзоры и мета-анализы сердечно-сосудистых заболеваний включали порядка 19 исследований [9]. Было установлено, что уровни систолического и диастолического артериального давления в группе детей, рожденных с применением ВРТ были выше, чем среди их сверстников, рожденных после спонтанной беременности. Наибольшие значения этих параметров были характерны для детей, рожденных в 1990-1999 гг. и не характерны для детей, рожденных после 2000 года. Согласно данным этого исследования в двух группах детей наблюдался сопоставимый индекс массы тела, уровень липопротеинов низкой плотности, холестерина и инсулина натощак.

По данным швейцарского исследования отмечается сосудистая дисфункция в группе детей, рожденных с применением ВРТ [10]. Данные нарушения сохранялись при повторном обследовании в возрасте 16-17 лет. Также у этих детей регистрировались более высокие значения систолического и диастолического давлений [11]. В целом, ограниченные данные свидетельствуют о потенциальном повышенном артериальном давлении у детей, рожденных с помощью ВРТ, а также о субоптимальной сердечно-сосудистой функции [12].

В отношении метаболических заболеваний имеется ряд публикаций, демонстрирующих противоречивые данные о риске развития сахарного диабета первого типа. В целом, отсутствуют доказательства, подтверждающие наличие такой ассоциации, но у детей, рожденных с применением ВРТ при наличии циклов заморозки/оттаивания риск возникновения сахарного диабета первого типа был достоверно выше [13].

Отмечается небольшое количество исследований, посвященных изучению риска развития заболеваний респираторной системы у детей, рожденных с применением ВРТ. Шведское исследование, включавшее 2628728 детей, рожденных в 1982-2007 годах, выявило повышенный риск развития астмы у детей, родившихся после ВРТ, увеличив абсолютный риск с 4,4 до 5,6\% [14]. Хотя возможный биологический механизм увеличенной частоты развития этих состояний, как и в случае других перинатальных исходов, достоверно не определен, некоторые утверждают, что наблюдаемые ассоциации могут быть объяснены субфертильностью матери, конфаундинг-факторами, изменяющими иммунитет матери во время беременности, такими как существовавшие ранее заболевания, включая астму и аллергию, или внешними факторами, такими как прием лекарственных препаратов и курение. Кроме того, было высказано предположение о том, что, поскольку женщины, проходящие процедуры ВРТ, как правило, имеют более высокий социально-экономический статус и большую массу тела при повышенной распространенности метаболических нарушений, их потомство может подвергаться повышенному риску неблагоприятных исходов. Таким образом, высокая распространенность метаболических нарушений в бесплодной популяции пациентов может оказывать долгосрочное воздействие по генетическим и эпигенетическим механизмам [15-17].

Ученые из Брюсселя опубликовали первые исследования репродуктивного здоровья у детей, рожденных с помощью ВРТ. Было обнаружено, что у мальчиков, рожденных после ИКСИ, качество спермы было значительно ниже, чем в контрольной группе. Однако не было выявлено 
четкой корреляции между параметрами спермы детей и их отцов $[18,19]$. Те же авторы показали сравнимые уровни половых гормонов, количество антральных фолликулов и уровень антимюллерова гормона у девочек, зачатых после ИКСИ [20]. В целом, ограниченные данные, опубликованные о репродуктивном здоровье у детей, рожденных с применением ВРТ, указывают на некоторое ухудшение количества сперматозоидов у мужчин, в то время как у женщин никаких неблагоприятных последствий выявлено не было.

Область репродуктивной медицины медленно продвигается от повышения эффективности проводимых мероприятий, к сосредоточению внимания на оптимизации здоровья потомства, рожденного с помощью этого метода. Проведение большего числа и более качественных исследований взаимосвязи между применением ВРТ и долгосрочными результатами в области здравоохранения имеет важнейшее значение [21]. Однако любой повышенный риск, наблюдаемый среди детей, рожденных после применения ВРТ, должен быть оценен критически [22]. Приведенные исследования часто не позволяют рассчитать абсолютные (а не только относительные) риски исследуемых заболеваний, подавляющее большинство детей, рожденных с помощью ВРТ, имеют такие же показатели здоровья, как и дети, рожденные в результате спонтанной беременности [23].

Исследования, изучающие влияние воздействия ВРТ на детство и долгосрочные последствия для здоровья потомства, сталкиваются с целым рядом проблем. Вопервых, субфертильность родителей потомства, по-видимому, повышает риск множественных проблем со здоровьем, независимо от того, было ли зачатие выполнено с медицинской помощью или нет. Инвазивность необходимого лечения, вероятно, увеличивает риск для потомства. Тем не менее, это не позволяет установить, является ли тяжесть субфертильного фенотипа или само ВРТ вмешательство причиной повышенного риска. Кроме того, трудно контролировать факторы, которые также могут повысить риск детской заболеваемости, такие как пересадка нескольких эмбрионов или преждевременные роды. Тем не менее, необходимо продолжать пристальное наблюдение за влиянием ВРТ на долгосрочный прогноз развития детей. Сложность в сборе таких данных заключается в том, что требуется определенное время для выявления связей между редкими событиями и воздействием ВРТ.

Представленные исследования позволяют сформировать общее представление о заболеваемости детей, рожденных после применения ВРТ. Вместе с тем, во всех исследованиях подчеркивается роль, которая отводится материнским факторам (возраст на момент наступления беременности, акушерский анамнез, социальное благополучие и др.). Имеются единичные исследования, направленных на изучение различий в здоровье детей, рожденных с применением ВРТ женщинами разного репродуктивного возраста. Принимая во внимание тот факт, что за медицинским лечением бесплодия обращаются, как правило, женщины позднего репродуктивного возраста, необходимо отдельное исследовать особенности формирования здоровья детей в данной группе женщин.

\section{Дополнительная информация}

Конфликт интересов. Автор декларирует отсутствие явных и потенциальных конфликтов интересов, о которых необходимо сообщить в связи с публикацией данной статьи.

\section{Литература}

1. Reigstad M.M., Oldereid N.B., Omland A.K., et al. Literature review on cancer risk in children born after fertility treatment suggests increased risk of haematological cancers // Acta Paediatrica. 2017. Vol. 106, №5. P. 698-709. doi:10.1111/ apa. 13755
2. Henningsen A.A., Gissler M., Skjaerven R., et al. Trends in perinatal health after assisted reproduction: a Nordic study from the CoNARTaS group // Human Reproduction. 2015. Vol. 30, №3. P. 710716. doi:10.1093/humrep/deu345

3. Källén B., Finnström O., Lindam A., et al. Trends in delivery and neonatal outcome after in vitro 
fertilization in Sweden: data for 25 years // Human Reproduction. 2010. Vol. 25, №4. P. 1026-1034. doi:10.1093/humrep/deq003

4. Kettner L.O., Henriksen T.B., Bay B., et al. Assisted reproductive technology and somatic morbidity in childhood: a systematic review // Fertility and Sterility. 2015. Vol. 103, №3. P. 707-719. doi:10.1016/j.fertnstert.2014.12.095

5. Choufani S., Shuman C., Weksberg R. BeckwithWiedemann syndrome // American Journal of Medical Genetics. Part C: Seminars in Medical Genetics. 2010. Vol. 154C, №3. P. 343-354. doi:10.1002/ajmg.c.30267

6. Williams C.L., Bunch K.J., Murphy M.F.G., et al. Cancer risk in children born after donor ART // Human Reproduction. 2018. Vol. 33, №1. P. 140-146. doi:10.1093/humrep/dex333

7. Williams C.L., Bunch K.J., Stiller C.A., et al. Cancer risk among children born after assisted conception // The New England Journal of Medicine. 2013. Vol. 369, №19. P. 1819-1827. doi:10.1056/ NEJMoa1301675

8. Bergh C., Wennerholm U.-B. Long-term health of children conceived after assisted reproductive technology // Upsala Journal of Medical Science. 2020. Vol. 125, №2. P. 152-157. doi:10.1080/03009734. 2020.1729904

9. Guo X., Liu X., Jin L., et al. Cardiovascular and metabolic profiles of offspring conceived by assisted reproductive technologies: a systematic review and meta-analysis // Fertility and Sterility. 2017. Vol. 107, №3. P. 622-631.e625. doi:10.1016/ j.fertnstert. 2016.12.007

10. Scherrer U., Rexhaj E., Allemann Y., et al. Cardiovascular dysfunction in children conceived by assisted reproductive technologies // European Heart Journal. 2015. Vol. 36, №25. P. 1583-1589. doi:10.1093/eurheartj/ehv145

11. Meister T.A., Rimoldi S.F., Soria R., et al. Association of assisted reproductive technologies with arterial hypertension during adolescence // Journal of the American College of Cardiology. 2018. Vol. 72, №11. P. 1267-1274. doi:10.1016/j.jacc.2018.06.060

12. Zandstra H., van Montfoort A.P.A., Dumoulin J.C.M., et al. Increased blood pressure and impaired endothelial function after accelerated growth in IVF/ICSI children // Human Reproduction Open. 2020. Vol. 2020, №1. P. hoz037. doi:10.1093/hropen/hoz037

13. Norrman E., Petzold M., Clausen T.D., et al. Type 1 diabetes in children born after assisted reproductive technology: a register-based national cohort study // Human Reproduction. 2020. Vol. 35, №1. P. 221-231. doi:10.1093/humrep/dez227

14. Källén B., Finnström O., Nygren K.G., et al. Asthma in Swedish children conceived by in vitro fertilization // Archives of Disease Childhood. 2013. Vol. 98, №2. P. 92-96. doi:10.1136/archdischild-2012-301822

15. Davies M. Infertility treatment at the edge: discovery and risk converge at the limits of knowledge // Archives of Disease Childhood. 2013. Vol. 98, №2.
P. 89-90. doi:10.1136/archdischild-2012-302487

16. Shiota K., Yamada S. Intrauterine environment-genome interaction and children's development (3): assisted reproductive technologies and developmental disorders // The Journal of Toxicological Science. 2009. Vol. 34, Suppl 2. P. SP287-291. doi:10.2131/jts.34.sp287

17. Guibas G.V., Moschonis G., Xepapadaki P., et al. Conception via in vitro fertilization and delivery by Caesarean section are associated with paediatric asthma incidence // Clinical and Experimental Allergy. 2013. Vol. 43, №9. P. 1058-1066. doi:10. 1111/cea.12152

18. Belva F., Bonduelle M., Roelants M., et al. Semen quality of young adult ICSI offspring: the first results // Human Reproduction. 2016. Vol. 31, №12. P. 2811-2820. doi:10.1093/humrep/dew245

19. Belva F., Roelants M., De Schepper J., et. al. Reproductive hormones of ICSI-conceived young adult men: the first results // Human Reproduction. 2017. Vol. 32, №2. P. 439-446. doi:10.1093/humrep/dew324

20. Belva F., Roelants M., Vloeberghs V., et al. Serum reproductive hormone levels and ultrasound findings in female offspring after intracytoplasmic sperm injection: first results // Fertility and Sterility. 2017. Vol. 107, №2. P. 934-939. doi:10.1016/j. fertnstert.2017.02.102

21. De Rycke M., Goossens V., Kokkali G., et al. ESHRE PGD Consortium data collection XIV-XV: cycles from January 2011 to December 2012 with pregnancy follow-up to October 2013 // Human Reproduction. 2017. Vol. 32, №10. P. 1974-1994. doi:10.1093/humrep/dex265

22. Fruchter E., Beck-Fruchter R., Hourvitz A., et al. Health and functioning of adolescents conceived by assisted reproductive technology // Fertility and Sterility. 2017. Vol. 107, №3. P. 774-780. doi:10.1016/j.fertnstert.2016.12.001

23. Seli E., Morin S. Assisted Reproductive Technology and Origins of Disease: The Clinical Realities and Implications // Seminars in Reproductive Medicine. 2018. Vol. 36, №3-04. P. 195-203. doi:10.1055/s-0038-1677048

\section{References}

1. Reigstad MM, Oldereid NB, Omland AK, et al. Literature review on cancer risk in children born after fertility treatment suggests increased risk of haematological cancers. Acta Paediatrica. 2017;106(5): 698-709. doi:10.1111/apa.13755

2. Henningsen AA, Gissler M, Skjaerven R, et al. Trends in perinatal health after assisted reproduction: a Nordic study from the CoNARTaS group. Human Reproduction. 2015;30(3):710-16. doi:10. 1093/humrep/deu345

3. Källén B, Finnström O, Lindam A, et al. Trends in delivery and neonatal outcome after in vitro fertilization in Sweden: data for 25 years. Human Reproduction. 2010;25(4):1026-34. doi:10.1093/humrep/ deq003

4. Kettner LO, Henriksen TB, Bay B, et al. Assisted reproductive technology and somatic morbidity 
in childhood: a systematic review. Fertility and Sterility. 2015;103(3):707-19. doi:10.1016/j.fertnstert. 2014.12.095

5. Choufani S, Shuman C, Weksberg R. BeckwithWiedemann syndrome. American Journal of Medical Genetics. Part C: Seminars in Medical Genetics. 2010;154C(3):343-54. doi:10.1002/ajmg.c.30267

6. Williams CL, Bunch KJ, Murphy MFG, et al. Cancer risk in children born after donor ART. Human Reproduction. 2017;33(1):140-6. doi:10.1093/hum rep/dex333

7. Williams CL, Bunch KJ, Stiller CA, et al. Cancer risk among children born after assisted conception. The New England Journal of Medicine. 2013;369 (19):1819-27. doi:10.1056/ NEJMoa1301675

8. Bergh C, Wennerholm U-B. Long-term health of children conceived after assisted reproductive technology. Upsala Journal of Medical Science. 2020: 125(2):152-7. doi:10.1080/03009734.2020.1729904

9. Guo X, Liu X, Jin L, et al. Cardiovascular and metabolic profiles of offspring conceived by assisted reproductive technologies: a systematic review and metaanalysis. Fertility and Sterility. 2017;107(3): 622-31.e625. doi:10.1016/j.fertnstert. 2016.12.007

10. Scherrer U, Rexhaj E, Allemann Y, et al. Cardiovascular dysfunction in children conceived by assisted reproductive technologies. European Heart Journal. 2015;36(25):1583-9. doi:10.1093/eur heartj/ehv145

11. Meister TA, Rimoldi SF, Soria R, et al. Association of assisted reproductive technologies with arterial hypertension during adolescence. Journal of the American College of Cardiology. 2018;72(11): 1267-74. doi:10.1016/j.jacc.2018.06.060

12. Zandstra H, van Montfoort APA, Dumoulin JCM, et al. Increased blood pressure and impaired endothelial function after accelerated growth in IVF/ICSI children. Human Reproduction Open. 2020;2020(1):hoz037. doi:10.1093/hropen/hoz037

13. Norrman E, Petzold M, Clausen TD, et al. Type 1 diabetes in children born after assisted reproductive technology: a register-based national cohort study. Human Reproduction. 2020;35(1):221-31. doi:10. 1093/humrep/dez227
14. Källén B, Finnström O, Nygren KG, et al. Asthma in Swedish children conceived by in vitro fertilization. Archives of Disease Childhood. 2013;98(2): 92-6. doi:10.1136/archdischild-2012-301822

15. Davies M. Infertility treatment at the edge: discovery and risk converge at the limits of knowledge. Archives of Disease Childhood. 2013;98(2):89-90. doi:10.1136/archdischild-2012-302487

16. Shiota K, Yamada S. Intrauterine environment-genome interaction and children's development (3): assisted reproductive technologies and developmental disorders. The Journal of Toxicological Sciences. 2009;34(suppl 2):SP287-91. doi:10.2131/jts.34.sp287

17. Guibas GV, Moschonis G, Xepapadaki P, et al. Conception via in vitro fertilization and delivery by Caesarean section are associated with paediatric asthma incidence. Clinical and Experimental Allergy. 2013;43(9):1058-66. doi:10.1111/cea.12152

18. Belva F, Bonduelle M, Roelants M, et al. Semen quality of young adult ICSI offspring: the first results. Human Reproduction. 2016;31(12):2811-20. doi:10.1093/humrep/dew245

19. Belva F, Roelants M, De Schepper J, et. al. Reproductive hormones of ICSI-conceived young adult men: the first results. Human Reproduction. 2017; 32(2):439-46. doi:10.1093/humrep/dew324

20. Belva F, Roelants M, Vloeberghs V, et al. Serum reproductive hormone levels and ultrasound findings in female offspring after intracytoplasmic sperm injection: first results. Fertility and Sterility. 2017; 107(2):934-9. doi:10.1016/j.fertnstert.2017.02.102

21. De Rycke M, Goossens V, Kokkali G, et al. ESHRE PGD Consortium data collection XIV-XV: cycles from January 2011 to December 2012 with pregnancy follow-up to October 2013. Human Reproduction. 2017;32(10):1974-94. doi:10.1093/humrep/dex265

22. Fruchter E, Beck-Fruchter R, Hourvitz A, et al. Health and functioning of adolescents conceived by assisted reproductive technology. Fertility and Sterility. 2017; 107(3):774-80. doi:10.1016/j.fertnstert.2016.12.001

23. Seli E, Morin S. Assisted Reproductive Techno-logy and Origins of Disease: The Clinical Realities and Implications. Seminars in Reproductive Medicine. 2018;36(3-04):195-203. doi:10.1055/s-0038-1677048

\section{Информация об авторе [Author Info]}

Кузьмичев Кирилл Александрович - аспирант кафедры общественного здоровья и здравоохранения ИПО, Самарский государственный медицинский университет, Самара, Российская Федерация. E-mail: kirill.kuzmichev@ yahoo.com SPIN: 7019-3053, ORCID ID: 0000-0002-5853-1838.

Kirill A. Kuzmichev - PhD-student of the Department of Public Health and Healthcare of PEI, Samara State Medical University, Samara, Russian Federation. E-mail: kirill.kuzmichev@yahoo.com SPIN: 7019-3053, ORCID ID: 0000-0002-5853-1838.

Цитировать: Кузьмичев К.А. Особенности заболеваемости детей, рожденных с помощью вспомогательных репродуктивных технологий // Наука молодых (Eruditio Juvenium). 2020. T. 8, №4. C. 629-634. doi:10.23888/HMJ202084629-634

To cite this article: Kuzmichev KA. Characteristics of morbidity of children born using assisted reproductive technologies. Science of the young (Eruditio Juvenium). 2020; 8(4):629-34. doi:10.23888/HMJ202084629-634

Поступила / Received: 02.03 .2020 Принята в печать / Accepted: 01.12.2020 